American Journal of Environmental Sciences 4 (4): 316-325, 2008

ISSN 1553-345X

(C) 2008 Science Publications

\title{
Performance Evaluation of a Waste Stabilization Pond in a Rural Area in Egypt
}

\author{
Mahassen M. El-Deeb Ghazy, Waled Morsy El-Senousy, \\ Azza. M. Abdel-Aatty and Mohammed Kamel \\ Department of Water Pollution Research, National Research Center, Cairo, Egypt
}

\begin{abstract}
The performance evaluation of the waste stabilization pond (WSP) as a model of domestic wastewater treatment unit in rural area was carried out. The unit comprised of anaerobic, facultative and maturation ponds in two series. The effluents of WSP which are discharged in the drain had the BOD reduced to $109-245 \mathrm{mg} \mathrm{L}^{-1}$ (Mean $=145.3 \mathrm{mg} \mathrm{L}^{-1}, 50.65 \%$ removal), while the COD was reduced to 221-400 $\mathrm{mg} \mathrm{L}^{-1}$ (Mean $=289 \mathrm{mg} \mathrm{L}^{-1}, 48.95 \%$ removal) and the total suspended solids (TSS) were reduced to $118-190 \mathrm{mg} \mathrm{L}^{-1}$ (Mean $=157.8 \mathrm{mg} \mathrm{L}^{-1}, 44.3 \%$ removal). The reduction percentages of total coliform (TC), faecal coliform (FC), E.coli, faecal streptococci (FS), salmonellae and Listeria were 98.8, 95.6, 79.4, 96.8, 97.9 and 89.5\% respectively. Also, the removal percentages of coliphage and infectious rotaviruses were 49.03 and $99.66 \%$ respectively. Identical sequences of rotaviruses VP-6 detected in the final effluent of the pond and the drain were observed. Euglena variables and Chlamydomonas reinhardii were predominant in anaerobic, facultative and maturation effluents. It has been noticed that pollution affected species diversity of zooplankton; the number of species in facultative pond was 8 species because of high pollution level, whereas in maturation pond increased to 21 species. Also, pollution in anaerobic pond increased density of ciliates (Protozoa) which are known to be bio-indicators of organic pollution. The percent removal of ciliates in the maturation pond was $70 \%$. It is recommended to make some modifications in the design to increase the efficiency of WSP.
\end{abstract}

Key words: zooplankton-protozoa-algae-bacteria-viruses

\section{INTRODUCTION}

The most appropriate wastewater treatment is that which will produce an effluent meeting the recommended microbiological and chemical quality guidelines both at low cost and with minimal operational and maintenance requirements. Different systems are used worldwide for wastewater treatment such as activated sludge, trickling filter and waste stabilization pond systems. Pond systems are commonly employed for municipal sewage purification, especially in developing countries, due to its costeffectiveness and high potential of removing different pollutants ${ }^{[3,6]}$.

WSPs are designed to achieve different forms of treatment up to three stages in series, depending on the organic strength of the input waste and effluent quality objectives. Usually, classical WSPs consist of an anaerobic pond, followed by primary or secondary facultative ponds. If further pathogen reduction is necessary, maturation ponds will be introduced to provide tertiary treatment. WSPs are very widely used for small rural communities but large systems exist in Mediterranean basin, France and also in Spain and
Portugal. However, in warmer climates (the Middle East, Africa, Asia and Latin America) ponds are commonly used for large populations ${ }^{[15]}$.

In developing countries and especially in the tropical and equatorial regions like Egypt, a shortage of wastewater treatment systems is observed in rural communities. There is a great need to wastewater treatment systems to avoid the health risk problems in these communities. Wastewater treatment by WSPs has been considered an ideal way of using natural processes to improve wastewater effluents. In natural treatment systems such as WSP, the pathogens are progressively removed along the pond series with the highest removal efficiency taking place in the maturation ponds ${ }^{[21]}$.

The aim of this study was to evaluate the performance of WSP in rural area in Egypt and to determine its role in the contamination of the drain.

\section{MATERIALS AND METHODS}

Wastewater treatment system in El-Mofti (Kafr ElSheikh, Egypt) was designed to serve 3000 persons. Wastewater flow is about $225 \mathrm{~m}^{3} /$ day mainly of domestic origin. This system consists of 500 primary

Corresponding Author: Waled Morsy El-Senousy, Water Pollution Department, National Research Center, Cairo, Egypt 
septic tanks (each septic tank has approximately volume $1.8 \mathrm{~m}^{3}$ with area $1.12 \mathrm{~m}^{2}$ and depth $1.6 \mathrm{~m}$ ) which used as primary treatment, a pumping station and wastewater stabilization pond which has two lines in parallel. Each line of pond consists of an anaerobic pond with volume $1400 \mathrm{~m}^{3}$ (depth $3 \mathrm{~m}$ and area 475 $\mathrm{m}^{2}$ ), a facultative pond with volume $1500 \mathrm{~m}^{3}$ (depth 1.5 $\mathrm{m}$ and area $1050 \mathrm{~m}^{2}$ ) and a maturation pond with volume $850 \mathrm{~m}^{3}$ (depth $1.4 \mathrm{~m}$ and area $635 \mathrm{~m}^{2}$ ).

Effluents of 500 septic tanks are collected and discharged to pump station which in turn is discharged to WSP. The final effluents of WSP are discharged into El-Sabahi agricultural drain.

Sampling sites: Wastewater and water samples were collected monthly during the period from may 2005 until February 2006 at seven sites from each stages of WSP and agricultural drain which receives the final effluents of WSP. Samples from 1-4 represent: 1-influent (effluent of all septic tanks), 2-anaerobic effluents, 3-facultative effluents and 4-maturation effluents. Samples no.5-7 represent: 5-drain before mixing with treated effluents, 6-mixing point and 7-after $700 \mathrm{~m}$ from mixing point in El-Sabahi drain which receives the final effluent of WSP.

All samples were collected and transported within ice box and analyzed within $6 \mathrm{~h}$ of collection for chemical and biological examinations.

\section{Samples analysis}

Physico-chemical analysis: Some physicochemical parameters such as temperature, $\mathrm{pH}$, total suspended solids (TSS), chemical oxygen demand (COD), biological oxygen demand (BOD) were determined according to $\mathrm{APHA}^{[2]}$ and phosphate according to Gales et al. $^{[13]}$.Additionally, nitrate was analyzed according to $\mathrm{DEV}^{[9]}$.

\section{Biological examination}

Algae: Algal growth was determined by measuring Chlorophyll a content Chl(a) spectrophotometrically and calculated according to $\mathrm{APHA}^{[2]}$. Identification of algal community structure was examined by identification keys ${ }^{[31,32]}$.

Zooplankton: For zooplankton identification, few samples were filtered through a net of $55 \mu \mathrm{m}$ pore size to concentrate zooplankton in $100 \mathrm{ml}$ of water but other samples containing great numbers of organisms were taken without filtration. Concentrated samples and nonfiltered samples were then preserved by Lugol's solution $^{[20]}$.
Zooplankton organisms were identified according to Edmondson ${ }^{[10]}$ and were counted microscopically in $1.5 \mathrm{ml}$ sub-samples in a Hawksley cell until attaining at least 60 individuals ${ }^{[23]}$

Bacteriological examination: Total bacterial count was determined using poured plate method while classical bacterial indicator (total coliform TC, faecal coliform FC, Escherishia coli (E.coli) and Faecal streptococci FC) were determined using MPN method. All parameters were carried out according to $\mathrm{APHA}^{[2]}$ except FC and E.coli. They were carried out according to Kamel ${ }^{[16]}$. Additionally, salmonellae and Listeria determination were carried out according to El-Taweel et al. ${ }^{[12]}$.

\section{Virological examination:}

Concentration of water and wastewater samples: All samples were concentrated by filtration through negatively charged nitrocellulose membranes according to Smith and Gerba ${ }^{[30]}$ and Rose et al. ${ }^{[28]}$. Then all samples were reconcentrated using an organic flocculation method according to Katzenelson et al. ${ }^{[18]}$.

Nucleic acid extraction: Nucleic acids were extracted using RNA viral extraction Kit (Qiagen) according to manufacturer's instructions.

Rotavirus detection using RT-PCR: The primers VP6-3 5-GCTTTAAAACGAAGTCTTCAAC-3 and VP6-4 5-GGTAAATTACCAATTCCTCCAG-3 were used for amplification of a fragment of the VP6-coding gene corresponding to nucleotides 2-187 for rotavirus with a predicted product size of $190 \mathrm{bp}^{[33]}$.

Sequencing of amplified products: RT-PCR products of selected samples were sequenced. Fifty to one hundred $\mu$ l of the RT-PCR products were purified using a high pure PCR products purification kit (Qiagen) following the manufacturer's instructions. Cycle sequencing was performed on 1 to $7 \mathrm{ml}$ of the purified products with an ABI prism Big dye termination cycle sequencing ready reaction kit (applied biosystem) using the same primers as in the PCR and following the manufacturer's instructions. The DNA was sequenced with an ABI prism 310 automated DNA sequencer.

Sequence data from both strands of the PCR products were aligned and compared by using the clustalw and blast programs (European bioinformatics institute).

Infection of CaCo-2 cells: Infection of $\mathrm{CaCo}-2$ cells was performed as previously described ${ }^{[26]}$. Briefly, after 30-min of preactivation with $10 \mu \mathrm{g}$ of trypsin/ml 
(grade IX; Sigma) at $37^{\circ} \mathrm{C}$, samples were 10 -fold diluted in PBS. Then, $100 \mu$ l of direct samples or dilutions were inoculated into $\mathrm{CaCo}-2$ monolayers grown in multiwell-plates (6 wells). After a $1 \mathrm{~h}$ adsorption, a serum free overlay medium $(3 \mathrm{ml})$ containing trypsin $(5 \mu \mathrm{g} / \mathrm{ml})$ was added and the cells were placed at $37^{\circ} \mathrm{C}$. Four days postinfection, cells were collected by centrifugation at $800 \mathrm{~g}$, resuspended in 300 $\mu l$ of PBS and freezed and thawed three times.

Detection of rotavirus infectious units using CC-RTPCR: Rotavirus cell culture RT-PCR (CC-RT-PCR) assay was performed on suspensions of infected $\mathrm{CaCo}-$ 2 cells. Primers VP6-3 and VP6-4 were used. RT-PCR method was the same as described previously. The detection limit in this tissue culture assay using $100 \mu \mathrm{l}$ of inoculation is $1 \mathrm{X} 10^{1} \mathrm{CC}-\mathrm{RT}$-PCR units $/ \mathrm{ml}$, (where CC-RT-PCR units is the reciprocal end point dilution detectable by CC-RT-PCR) ${ }^{[1]}$.

\section{RESULTS}

In this study, septic tanks were used as a pretreatment of house holds wastewater. The overall flow of wastewater to WSP which is the effluent of the septic tanks was $225 \mathrm{~m}^{3} /$ day. The water temperature records were between $18{ }^{\circ} \mathrm{C}$ and $29^{\circ} \mathrm{C}$, the average water temperature in anaerobic and facultative was $23.4^{\circ} \mathrm{C}$ while in maturation pond was $21.2^{\circ} \mathrm{C}$. The average removal efficiencies of organic load in WSP measured as COD were 28.9, 20.24 and 9.9\% after anaerobic, facultative and maturation ponds respectively. The anaerobic effluent indicated a BOD average value of $229 \mathrm{mg} \mathrm{L}^{-1}$, the facultative effluents $180.7 \mathrm{mg} \mathrm{L}^{-1}$ and maturation effluents $145.3 \mathrm{mg} \mathrm{L}^{-1}$. The removal efficiencies of this parameter were 22 ,
21.1 and $19.6 \%$ in anaerobic, facultative and maturation effluents respectively Table 1 . The mean values of TSS in this system were $283.3 \mathrm{mg} \mathrm{L}^{-1}, 214.3 \mathrm{mg} \mathrm{L}^{-1}, 176.3$ $\mathrm{mg} \mathrm{L}^{-1}$ and $157.8 \mathrm{mg} \mathrm{L}^{-1}$ in influent, anaerobic , facultative, maturation effluents and the reduction of TSS was $24.4,17.7$ and $10.5 \%$ in anaerobic, facultative and maturation ponds respectively. The overall reduction of dissolved phosphorus and nitrate were 51.4 and $55.5 \%$ respectively. The characteristic properties of drain before discharge revealed that $\mathrm{pH}$ was 7.8 while, COD, BOD and TSS were $199.3 \mathrm{mg} \mathrm{L}^{-1}, 101.5 \mathrm{mg} \mathrm{L}^{-1}$ and $152 \mathrm{mg} \mathrm{L}^{-1}$ respectively. The concentration of these parameters were increased after discharge the WSP effluents to the drain where, $\mathrm{pH}$ was 7.9 while $\mathrm{COD}$, BOD and TSS were $276 \mathrm{mg} \mathrm{L}^{-1}, 143 \mathrm{mg} \mathrm{L}^{-1}$ and 161 $\mathrm{mg} \mathrm{L}^{-1}$ respectively. After $700 \mathrm{~m}$ of discharge point $\mathrm{pH}$ was 7.5 and COD, BOD and TSS were $101 \mathrm{mg} \mathrm{L}^{-1}, 49$ $\mathrm{mg} \mathrm{L}^{-1}$ and $41 \mathrm{mg} \mathrm{L}^{-1}$.

The mean and removal percentages of microbial indicators and bacterial pathogens for each stage of WSP and each of the three points of the drain receiving the WSP effluent are presented in Table (2). The results showed that the load of bacterial-indicators with influent of WSP samples were $10^{12}-10^{13} \mathrm{Cfu} / \mathrm{ml}$ for total bacterial count, $10^{12}$ for $\mathrm{TC}, 10^{10}$ for $\mathrm{FC}, 10^{8}$ for $\mathrm{E}$. coli and $10^{8}$ for $\mathrm{FS}$ as $\mathrm{Mpn} / 100 \mathrm{ml}$. Also, coliphage count was $1.49 \times 10^{2} \mathrm{Cfu} / 100 \mathrm{ml}$ and the bacterial pathogens of influent sample were $4.3 \times 10^{4}$ and $1.3 \times 10^{6}$ $\mathrm{Cfu} / 100 \mathrm{ml}$ for salmonellae and Listeria respectively. The average removal efficiencies of bacterial loads with anaerobic pond were 1-2 $\log _{10}$ unit (88.4-94.4\%) for total bacterial counts, one $\log _{10}$ unit for both $\mathrm{TC}$ $(96.2 \%)$ and $\mathrm{FC}(94.3 \%)$ and $2 \log _{10}$ units for both $\mathrm{E}$. coli $(98.5 \%)$ and FS $(98.9 \%)$.

Table 1: Some physico-chemical characteristics of wastewater samples (standard deviation is in between brackets)

\begin{tabular}{|c|c|c|c|c|c|c|c|}
\hline \multirow[b]{2}{*}{$\begin{array}{l}\text { Sampling } \\
\text { sites }\end{array}$} & \multicolumn{7}{|l|}{ Parameters } \\
\hline & $\begin{array}{l}\text { Nitrate } \mathrm{NO}^{3}-\mathrm{N} \\
\mathrm{mg} \mathrm{L}^{-1}\end{array}$ & $\begin{array}{l}\text { Dissolved phosphate } \\
\text { PO }^{4}-\mathrm{P} \mathrm{mg} \mathrm{L}^{-1}\end{array}$ & $\begin{array}{l}\text { Total phosphate } \\
\mathrm{PO}_{4}-\mathrm{P} \mathrm{mg} \mathrm{L}^{-1}\end{array}$ & $\begin{array}{l}\text { Biological oxygen } \\
\text { demand } \mathrm{mg} \mathrm{O}_{2} \mathrm{~L}^{-1}\end{array}$ & $\begin{array}{l}\text { Chemical oxygen } \\
\text { demand } \mathrm{mg} \mathrm{O}_{2} \mathrm{~L}^{-1}\end{array}$ & $\begin{array}{l}\text { Total suspended } \\
\text { solids } \mathrm{mg} \mathrm{L}^{-1}\end{array}$ & $\mathrm{~d} \mathrm{pH}$ \\
\hline \multicolumn{8}{|c|}{ WSP influents } \\
\hline Range & $0.81-1.01$ & $11-18$ & $14-26$ & $204-420$ & $445-782$ & $196-370$ & $7.7-8.2$ \\
\hline Mean & $0.91(0.15)$ & $14.4(2.6)$ & $20.1(4.5)$ & $294.4(85.9)$ & $566.1(117.2)$ & $283.3(65.7)$ & $7.89(0.184)$ \\
\hline \multicolumn{8}{|c|}{ Anaerobic effluents } \\
\hline Range & $0.34-0.69$ & $9-15$ & $12-25$ & $165-425$ & $239-750$ & $116-360$ & $7.74-8.2$ \\
\hline Mean & $0.51(0.15)$ & $11.6(2.4)$ & $16.7(4.2)$ & $229(94.5)$ & $402.5(178.0)$ & $214.3(92.3)$ & $8.019(0.182)$ \\
\hline \multicolumn{8}{|c|}{ Facultative effluents } \\
\hline Range & $0.25-0.65$ & $5.5-12$ & $8-13$ & $138-267$ & $207-507$ & $80-300$ & $7.7-8.6$ \\
\hline Mean & $0.384(0.163)$ & $7.9(2.2)$ & $11.1(1.7)$ & $180.7(48.4)$ & $321(114.9)$ & $176.3(90.2)$ & $8.26(0.23)$ \\
\hline \multicolumn{8}{|c|}{ Maturation effluents } \\
\hline Range & $0.17-0.67$ & $4-11$ & $7-13$ & $109-245$ & $221-400$ & $118-190$ & $7.9-9.0$ \\
\hline Mean & $0.39(0.15)$ & $7(2.4)$ & $10.5(2.3)$ & $145.3(46.3)$ & $289(69.2)$ & $157.8(30.9)$ & $8.34(0.40)$ \\
\hline
\end{tabular}



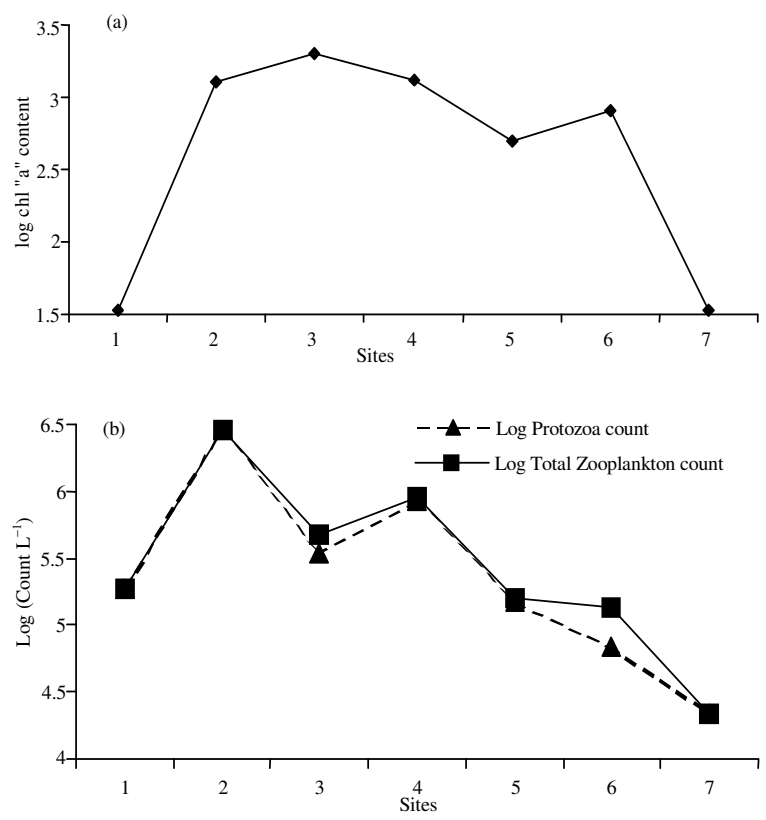

Fig. 1: Correlation between Chlorophyll "a" Concentrations (a) and Zooplankton Counts (b) at Different Sites of Operational Steps in ElMofti Stabilization Pond and Drain
Removal efficiency of coliphage was $34.7 \%$. The removal efficiencies in facultative and maturation ponds were more than $95 \%$ for classical bacterial indicators except total bacterial counts at $37^{\circ} \mathrm{C}(90 \%)$ and $E$. coli $(79.4 \%)$ in maturation pond. The removal of coliphage was $36.9 \%$ in facultative pond and $49.03 \%$ in maturation pond. Bacterial pathogens reductions were 85.3, 96.2, 97.7\% for salmonellae and 62.3, 84.5 and $89.5 \%$ for Listeria in anaerobic, facultative and maturation ponds respectively (Table 2). The microbial loads of water drain at mixing point were higher than the microbial loads before mixing point and considerable decrease in the loads was observed at 700 $\mathrm{m}$ from mixing point (Table 2). The mean count of Listeria was $59.11 \mathrm{Cfu} / 100 \mathrm{ml}$ but salmonellae was absent at $700 \mathrm{~m}$ from the mixing point in the drain.

Change in Chl (a) content of the studied wastewater during the various treatment stages is presented in Fig. 1. Available data revealed an increase in $\mathrm{Chl}$ (a) content of the raw wastewater $\left(33.8 \mu \mathrm{g} \mathrm{L}^{-1}\right)$ as it passes from the anaerobic to the facultative ponds which amounted to $1261.6 \mu \mathrm{g} \mathrm{\textrm {L } ^ { - 1 }}$ and $1833 \mu \mathrm{g}$ $\mathrm{L}^{-1}$ respectively, in maturation pond $\mathrm{Chl}(\mathrm{a})$ was decreased. The successive changes in algal community as the wastewater flow from the anaerobic to the

Table 2: Microbiological characteristics of wastewater stabilization pond and drain water receiving final effluent of pond

\begin{tabular}{|c|c|c|c|c|c|c|c|c|c|}
\hline \multirow{2}{*}{$\begin{array}{l}\text { Sampling } \\
\text { site }\end{array}$} & \multicolumn{2}{|c|}{ Total bacterial count $\mathrm{Cfu} / \mathrm{ml}$} & \multicolumn{3}{|c|}{ MPN-index/100ml } & \multirow{2}{*}{ FS } & \multirow{2}{*}{$\begin{array}{l}\text { Coliphage } \\
\text { Cfu/100 ml }\end{array}$} & \multicolumn{2}{|c|}{ Pathogenic bacteria $\mathrm{Cfu} / 100 \mathrm{ml}$} \\
\hline & At $37^{\circ} \mathrm{c}$ & At $22^{\circ} \mathrm{c}$ & $\mathrm{TC}$ & $\mathrm{FC}$ & E.coli & & & Salmonellae & Listeria \\
\hline \multicolumn{10}{|c|}{ WSP influents } \\
\hline Mean & $1.4 \times 10^{13}$ & $4.9 \times 10^{12}$ & $3.4 \times 10^{12}$ & $6.9 \times 10^{10}$ & $5.2 \times 10^{8}$ & $2.3 \times 10^{9}$ & 149.4 & $4.3 \times 10^{4}$ & $1.3 \times 10^{4}$ \\
\hline S.D & $36 \times 10^{13}$ & $6.7 \times 10^{12}$ & $5.6 \times 10^{12}$ & $6.6 \times 10^{10}$ & $7.6 \times 10^{8}$ & $3.9 \times 10^{9}$ & 38.04 & $6.1 \times 10^{4}$ & $1.5 \times 10^{4}$ \\
\hline \multicolumn{10}{|c|}{ Anaerobic effluents } \\
\hline Mean & $7.9 \times 10^{11}$ & $5.7 \times 10^{11}$ & $1.3 \times 10^{11}$ & $3.9 \times 10^{9}$ & $7.9 \times 10^{6}$ & $2.4 \times 10^{7}$ & 97.6 & $6.3 \times 10^{3}$ & $4.9 \times 10^{3}$ \\
\hline $\mathrm{R} \%$ & 94.4 & 88.4 & 96.2 & 94.3 & 98.5 & 98.9 & 34.7 & 85.3 & 62.3 \\
\hline S.D & $2.4 \times 0^{12}$ & $1.4 \times 10^{12}$ & $3.6 \times 10^{11}$ & $7 \times 10^{10}$ & $1.5 \times 10^{7}$ & $7.3 \times 10^{7}$ & 16.9 & $77 \times 10^{3}$ & $6.8 \times 10^{3}$ \\
\hline \multicolumn{10}{|c|}{ Facultative effluents } \\
\hline Mean & $7.9 \times 10^{9}$ & $1.1 \times 10^{10}$ & $3.6 \times 10^{8}$ & $3.6 \times 10^{7}$ & $3.6 \times 10^{5}$ & $8.1 \times 10^{5}$ & 61.6 & $2.4 \times 10^{2}$ & $7.6 \times 10^{2}$ \\
\hline $\mathrm{R} \%$ & 99 & 98.1 & 99.7 & 99.1 & 95.4 & 96.6 & 36.9 & 96.2 & 84.5 \\
\hline S.D & $1.9 \times 10^{10}$ & $2.8 \times 10^{10}$ & $9.2 \times 10^{8}$ & $3.6 \times 10^{7}$ & $4.1 \times 10^{5}$ & $1.4 \times 10^{6}$ & 8.8 & $4.2 \times 10^{2}$ & $1.1 \times 10^{3}$ \\
\hline \multicolumn{10}{|c|}{ Maturation effluents } \\
\hline Mean & $7.9 \times 10^{8}$ & $2 \times 10^{7}$ & $3.8 \times 10^{6}$ & $1.6 \times 10^{6}$ & $7.4 \times 10^{4}$ & $2.6 \times 10^{4}$ & 31.4 & 4.9 & $1.1 \times 10^{2}$ \\
\hline $\mathrm{R} \%$ & 90 & 99.8 & 98.8 & 95.6 & 79.4 & 96.8 & 49.03 & 97.9 & 89.5 \\
\hline S.D & $7.3 \times 10^{9}$ & $2.8 \times 10^{7}$ & $7.3 \times 10^{6}$ & $3.9 \times 10^{6}$ & $1.4 \times 10^{5}$ & $4.9 \times 10^{4}$ & 6.5 & 14.7 & 91.3 \\
\hline \multicolumn{10}{|c|}{ Drain water before mixing } \\
\hline Mean & $2.9 \times 10^{6}$ & $9.9 \times 10^{6}$ & $2.5 \times 10^{6}$ & $3 \times 10^{4}$ & $3.2 \times 10^{3}$ & $1.2 \times 10^{4}$ & 11.8 & 2 & 81.8 \\
\hline S.D & $4.8 \times 10^{6}$ & $2.4 \times 10^{7}$ & $4.8 \times 10^{6}$ & $3.9 \times 10^{4}$ & $7.1 \times 10^{3}$ & $3.1 \times 10^{4}$ & 2.9 & 6 & 87.4 \\
\hline \multicolumn{10}{|c|}{ Mixing point } \\
\hline Mean & $4 \times 10^{8}$ & $3.6 \times 10^{8}$ & $6.4 \times 10^{7}$ & $9.8 \times 10^{6}$ & $7.6 \times 10^{4}$ & $7.1 \times 10^{5}$ & 20.8 & 18.9 & $2.7 \times 10^{2}$ \\
\hline S.D & $8.1 \times 10^{8}$ & $6.1 \times 10^{8}$ & $1.7 \times 10^{8}$ & $2.2 \times 10^{7}$ & $1.6 \times 10^{5}$ & $1.5 \times 10^{6}$ & 2.3 & 53.01 & $2.4 \times 10^{2}$ \\
\hline \multicolumn{10}{|c|}{$700 \mathrm{~m}$ after mixing point } \\
\hline Mean & $1.1 \times 10^{7}$ & $4.4 \times 10^{6}$ & $6.7 \times 10^{5}$ & $2.7 \times 10^{5}$ & $6 \times 10^{2}$ & $1 \times 10^{4}$ & 9.4 & 0 & 59.11 \\
\hline S.D & $2.3 \times 10^{7}$ & $6.3 \times 10^{6}$ & $1.5 \times 10^{6}$ & $7.9 \times 10^{5}$ & $1.5 \times 10^{3}$ & $2.2 \times 10^{4}$ & 2.9 & 0 & 82.11 \\
\hline
\end{tabular}


Am. J. Environ. Sci., 4 (4): 316-325, 2008

Table 3: Change in community structure of algae in El-Mofti WSP

\begin{tabular}{|c|c|c|c|c|c|c|c|}
\hline \multirow[t]{3}{*}{ Algal taxa } & \multicolumn{5}{|c|}{ Sampling site } & & \\
\hline & \multirow[t]{2}{*}{ Raw } & \multirow{2}{*}{$\begin{array}{l}\text { Anaerobic } \\
- \text { Start }\end{array}$} & \multirow[b]{2}{*}{ End } & \multicolumn{2}{|c|}{ Facultative } & \multicolumn{2}{|c|}{ Maturation } \\
\hline & & & & Start & End & Start & End \\
\hline \multicolumn{8}{|l|}{ Green algae } \\
\hline Euglena variabilis & \pm & ++++ & + & +++ & ++ & + & ++ \\
\hline Chlamydomonas reinhardii & \pm & ++++ & +++ & +++ & +++ & ++ & +++ \\
\hline Cryptomonas erosa & \pm & ++++ & + & + & - & + & - \\
\hline Pandorina morium & \pm & \pm & - & \pm & - & \pm & - \\
\hline Phacus triquetre & \pm & \pm & + & ++ & ++ & + & ++ \\
\hline Haematococcus pluvialis & \pm & \pm & + & \pm & \pm & \pm & + \\
\hline Microactinum pusillum & \pm & \pm & ++ & + & ++ & \pm & + \\
\hline Siderocelis elegans & \pm & - & - & \pm & \pm & - & - \\
\hline Pediastrum clathatum & \pm & - & - & \pm & $\overline{ \pm}$ & - & - \\
\hline \multicolumn{8}{|l|}{ Blue-green algae } \\
\hline Oscillatoria limnetica & \pm & \pm & \pm & \pm & \pm & \pm & \pm \\
\hline Oscillatoria chlorine & \pm & \pm & \pm & \pm & \pm & \pm & \pm \\
\hline
\end{tabular}

maturation ponds are given in Table 3. In influent 9 green algal species found in rare numbers, also 2 species from blue green algae and diatoms group are not represented. The anaerobic pond was almost dominated by Euglena variabilis, Chlamydomonas reinhardii, Cryptomonas erosa, however in the facultative and maturation pond, algal community was represented by Euglena variabilis, Chlamydomonas reinhardii, Phacus triquetre. A pronounced change in Chl (a) value had occurred, $503 \mu \mathrm{g} \mathrm{L}{ }^{-1}$ before discharge and $816 \mu \mathrm{g} \mathrm{L}^{-1}$ at mixing point. As a result of dilution factor Chl (a) content become $33.5 \mu \mathrm{g} \mathrm{L}^{-1}$ in the drain after 700 meters. Available results revealed that Phacus triquetre, Euglena variabilis, Chlamydomonas reinhardii and Microactinum pusillum were detectable at the discharge point. After discharge by 700 meters, where the organic load diluted and the previous species were found in rare count beside the following species which represented the three algal groups: Diatoms, green algae and blue green algae. Diatoms were represented by Diatoma elongatum, Gryosigma attenuatum, Fragillaria capunica and Synedra ulna, green algae were represented by Scenedesmus quadricauda and and blue green algae were represented by Oscillatoria limnetica, Oscillatoria chlorine.

Zooplankton communities of this study are presented in Table 4a and 4b. Zooplankton groups were usually identified to the genus level except Copepoda that was identified to the suborder level (namely, Calanoida, Cyclopoida and Harpacticoida). In influent, protozoans especially ciliates dominated zooplankton biomass (count/liter) of which Didinium $\left(1380 \times 10^{2} /\right.$ liter) was the most abundant genus followed by less numbers of Astylozoon. Other zooplankton groups detected in influent were rotifers represented by
Philodina at 280/liter, crustaceans represented by the ostracod Cyprinotus at 140/liter, larval stages of insects at 540/liter and larval stages of Nematoda at 400/liter. In the anaerobic pond, the total zooplankton count was at its maximum $\left(28736 \times 10^{2} /\right.$ liter $)$. In that site the phylum Protozoa was also the predominant group because of the dominancy of the genus Stombidium (20400x $10^{2} /$ liter) which was followed by Aristerostoma at $6030 \times 10^{2} /$ liter. The phylum Rotatoria represented by Brachionus showed mean count 140/liter and there were no representatives of the two groups Arthropoda and Nematoda. The facultative pond was dominated by phylum Protozoa followed by rotifers and as in the anaerobic pond, representatives of arthropods and nematodes were not detected. The ciliate Aristerostoma $\left(2254 \times 10^{2} /\right.$ liter) was the dominant genus followed by Astylozoon at $673 \times 10^{2} /$ liter and by less number of Paramecium at $460 \times 10^{2} /$ liter. As we proceed from the facultative pond to the maturation pond, the mean count of zooplankton increased from $4720 \times 10^{2} /$ liter to $8810 \times 10^{2} /$ liter. The ciliates Dysteria, Tintinnopsis, Astylozoon and Mesodinium appeared in the wastewater samples taken from the maturation pond at mean counts of $1028 \times 10^{2}, 760 \times 10^{2}, 208 \times 10^{2}$ and $178 \times 10^{2}$ organisms/liter respectively and were preceded by the abundance of Aristerostoma at $6000 \times 10^{2} /$ liter. The rotifer Asplanchna was the dominant genus in the phylum Rotatoria and its count decreased from $1140 \times 10^{2} /$ liter in the facultative pond to $217 \times 10^{2} /$ liter in the maturation pond. The Zooplankton counts decreased gradually in the water samples as passing from maturation pond to the discharge point, then after mixing with the agricultural drain until reached to their minimum $\left(220 \times 10^{2} /\right.$ liter $)$ at $700 \mathrm{~m}$ from the mixing point with the drain. 
Am. J. Environ. Sci., 4 (4): 316-325, 2008

Table 4a: Zooplankton counts (Mean \pm SD) per liter in the wastewater samples taken from the treatment steps of the oxidation pond. Sites

Zooplankton

Influents

Anaerobic effluents Facultative effluents Maturation effluents

Phylum: Protozoa

Class:Rhizopoda and Actinopoda

Plagiopyxis

Amoeba

Arcella

Class: Ciliata

Vorticella

Paramecium

Mesodinium

Opercularia

Aristerostoma

Astylozoon

Acropisthium

Eschaneustyla

Podophrya

Aspidisca

Tintinnopsis

Coleps

Didinium

Chilodonella

Condylostoma

Thecacinata

Stombidium.

Dysteria

Frontonia

Tetrahymena

Class: Zooflagellata

Paramastix

Phylum: Rotatoria Class: Rotifera

Brachionus

Keratella

Lepadella

Philodina

Asplanchna

Asplanchnopus

Enteroplea

Manfredium

Polyarthra

Platyias

Trichotria

Diplois

Phylum: Arthropoda Class: Crustacea

Subclass:Branchiopoda

Order: Cladocera

Bosmina

Alona

Moinodaphnia

Subclass: Copepoda

Cyclopoids

Harpacticoids

Nauplius larva

Subclass: Ostracoda

$$
\text { Cyprinotus }
$$

Class: Insecta Larval Stages

Phylum: Nematoda Larval stages

Total Count /L

$5.4 \pm 7.79$
-
-
-

-

$21.4 \pm 39.39$

$45.4 \pm 90.8$

$384 \pm 768$

$-$

$-$

-

$1380 \pm 2760$

-

$-$

-

$-$

$\begin{array}{ll}497.4 \pm 951.9 & 82 \pm 164 \\ - & - \\ - & - \\ - & -\end{array}$

$1.4 \pm 2.8$

$5.4 \pm 10.8$

480. \pm 845.13

$6030 \pm 11890.7$

$1204 \pm 1497.22$

$122 \pm 178.71$

-

$-$

$-$

$-$

-

$-$

$-$

$204000 \pm 40800$

-

$-$

$1.4 \pm 2.8$

$-$

$-$

$-$

$-$

$-$

$-$

$-$

$-$

$-$

$-$

$-$

$-$

-

$-$

$$
-
$$$$
-
$$

$460 \pm 920$

$673.4+831.14$

$-$

$760 \pm 1520$

-

$40 \pm 80$

$1028 \pm 2056$

$-$

$1.4 \pm 2.8$

$38.6 \pm 21.2$

$23.8 \pm 20.76$

$1.4 \pm 2.8$

$10.8 \pm 13.23$

$216.6 \pm 426.73$

$1140 \pm 2280$

$57.4 \pm 114.8$

$1.4 \pm 2.8$

$1.4 \pm 2.8$

$1.4 \pm 2.8$

-

$-$
$2.6 \pm 5.2$

$1.4 \pm 2.8$

-

$1.4 \pm 2.8$

$4 \pm 8$

$2.6 \pm 5.2$

$8809.6 \pm 11324.37$

N.B.: All zooplankton counts are divided by $10^{2}$ 
Am. J. Environ. Sci., 4 (4): 316-325, 2008

Table 4b: Zooplankton counts (Mean \pm SD) per liter in the water samples taken from El Sabahi Drain

\begin{tabular}{|c|c|c|c|}
\hline \multirow[b]{2}{*}{ Zooplankton } & \multicolumn{3}{|l|}{ Sites } \\
\hline & Drain water before mixing & Mixing point & $\begin{array}{l}\text { After } 700 \text { meters of mixing } \\
\text { point }\end{array}$ \\
\hline \multicolumn{4}{|l|}{ Phylum: Protozoa } \\
\hline \multicolumn{4}{|l|}{ Class:Rhizopoda and Actinopoda } \\
\hline Centropyxis & $10.6 \pm 13.62$ & $16.28 \pm 28.63$ & - \\
\hline Plagiopyxis & 5.410 .8 & $16 \pm 32$ & $30 \pm 51.96$ \\
\hline Amoeba & - & $4 \pm 8$ & - \\
\hline Arcella & - & $1.4 \pm 2.8$ & - \\
\hline \multicolumn{4}{|l|}{ Class: Ciliata } \\
\hline Vorticella & - & $14.62 \pm 29.19$ & - \\
\hline Paramecium & - & - & $1.75 \pm 3.03$ \\
\hline Mesodinium & $245.4 \pm 477.41$ & $0.146 \pm 0.292$ & $120.78 \pm 201.69$ \\
\hline Opercularia & - & - & - \\
\hline Aristerostoma & - & - & - \\
\hline Astylozoon & $18 \pm 36$ & - & - \\
\hline Acropisthium & - & $0.146 \pm 0.168$ & $0.08 \pm 0.14$ \\
\hline Eschaneustyla & - & $0.084 \pm 0.168$ & $1.75 \pm 3.03$ \\
\hline Podophrya & $1.4 \pm 2.8$ & - & - \\
\hline Aspidisca & $4 \pm 8$ & $102 \pm 2.4$ & $52.5 \pm 9093$ \\
\hline Tintinnopsis & - & - & - \\
\hline Coleps & $5.4 \pm 10.8$ & $5.4 \pm 10.8$ & $3.25 \pm 5.63$ \\
\hline Didinium & - & - & - \\
\hline Chilodonella & - & $0.124 \pm 0.248$ & - \\
\hline Condylostoma & - & - & - \\
\hline Thecacinata & $1180 \pm 2360$ & - & - \\
\hline Stombidium. & - & - & - \\
\hline Dysteria & - & - & - \\
\hline Frontonia & - & $9.4 \pm 18.8$ & - \\
\hline Tetrahymena & - & $506 \pm 1012$ & - \\
\hline \multicolumn{4}{|l|}{ Class: Zooflagellata } \\
\hline Paramastix & $12 \pm 24$ & $20 \pm 40$ & $5 \pm 8.66$ \\
\hline \multicolumn{4}{|l|}{ Phylum: Rotatoria Class: Rotifera } \\
\hline Brachionus & $62.6 \pm 118.81$ & $62.62 \pm 97.98$ & - \\
\hline Keratella & $0.6 \pm 1.2$ & - & $0.08 \pm 0.14$ \\
\hline Lepadella & $2 \pm 4$ & - & - \\
\hline Philodina & $34.6 \pm 69.2$ & $18.7 \pm 27.56-$ & $1.75 \pm 3.03$ \\
\hline Asplanchna & - & $5.4 \pm 10.8$ & - \\
\hline Asplanchnopus & - & $568 \pm 1136$ & - \\
\hline Enteroplea & - & - & - \\
\hline Manfredium & - & - & - \\
\hline Polyarthra & - & - & - \\
\hline Platyias & $1.4 \pm 2.8$ & - & - \\
\hline Trichotria & $1.4 \pm 2.8$ & - & - \\
\hline Diplois & - & $0.02 \pm 0.04$ & - \\
\hline \multirow{2}{*}{\multicolumn{4}{|c|}{$\begin{array}{c}\text { Phylum: Arthropoda Class: Crustacea } \\
\text { Subclass:Branchiopoda } \\
\text { Order: Cladocera }\end{array}$}} \\
\hline & & & \\
\hline Bomina & - & $0.02 \pm 0.04$ & - \\
\hline Alona & - & - & - \\
\hline Moinodaphnia & - & $0.02 \pm 0.04$ & - \\
\hline \multicolumn{4}{|l|}{ Subclass: Copepoda } \\
\hline Cyclopoids & $0.6 \pm 1.2$ & - & - \\
\hline Harpacticoids & - & - & - \\
\hline Nauplius larva & $4.8 \pm 6.68$ & $0.02 \pm 0.04$ & $1.75 \pm 3.03$ \\
\hline \multicolumn{4}{|l|}{ Subclass: Ostracoda } \\
\hline Cyprinotus & - & - & - \\
\hline Class: Insecta Larval Stages & $0.6 \pm 1.2$ & - & - \\
\hline Phylum: Nematoda Larval stages & $6 \pm 12$ & $1.4 \pm 2.8$ & - \\
\hline Total Count /L & $1596.8 \pm 2756.10$ & $1351.54 \pm 2056.64$ & $220.5 \pm 212.55$ \\
\hline
\end{tabular}


Am. J. Environ. Sci., 4 (4): 316-325, 2008

Table 5: Number of infectious particles of rotaviruses per liter in the WSP treatment steps and drain water samples

\begin{tabular}{|c|c|c|c|c|c|c|c|c|}
\hline \multirow[t]{2}{*}{ Samples } & \multicolumn{8}{|c|}{ No. of rotavirus infectious particles (CC-RT-PCR units/liter) } \\
\hline & May & June & July & August & September & December & January & February \\
\hline & 2005 & 2005 & 2005 & 2005 & 2005 & 2005 & 2006 & 2006 \\
\hline WSP influents & --- & --- & --- & 0 & 0 & $1 \times 10^{4}$ & $1 \times 10^{6}$ & $1 \times 10^{4}$ \\
\hline Anaerobic effluents & --- & --- & --- & --- & --- & $1 \times 10^{3}$ & $1 \times 10^{4}$ & $1 \times 10^{2}$ \\
\hline Facultative effluents & --- & --- & --- & --- & --- & $1 \times 10^{2}$ & $1 \times 10^{3}$ & $1 \times 10$ \\
\hline Maturation effluents & --- & --- & --- & --- & --- & $1 \times 10^{2}$ & $1 \times 10^{2}$ & 1 \\
\hline Drain water before mixing & --- & --- & --- & --- & --- & $1 \times 10$ & 0 & --- \\
\hline Mixing point & --- & --- & --- & --- & --- & --- & $1 \times 10$ & --- \\
\hline After 700 meters of mixing point & --- & --- & --- & --- & --- & --- & 1 & --- \\
\hline
\end{tabular}

Rotaviruses were detected in 5 influent, 3 anaerobic, 3 facultative and 3 maturation effluent samples out of 8 samples for each stage using RT-PCR. Also, rotaviruses were detected two times before mixing and one time at both mixing point and after 700 $\mathrm{m}$ from mixing point of drain water samples. Sequence analysis of RT-PCR products revealed that they were belonged to rotavirus VP6. On the other hand, Table (5) showed the number of rotaviral infectious particles in the positive rotavirus VP6 samples. It can be observed that the number of infectious units ranged from 0 to $10^{6}, 10^{2}$ to $10^{4}$ and 10 to $10^{3}$ and from 1 to $10^{2}$ CC-RTPCR units/liter in influent, anaerobic effluent, facultative effluent and maturation effluent samples respectively. The count of infectious particles in water drain samples were 10, 10 and 1 CC-RT-PCR units/liter before mixing point, at mixing point and after $700 \mathrm{~m}$ from mixing point samples respectively. Sequence analysis revealed that identical sequences of rotavirus between influent, after anaerobic pond, after facultative pond, after maturation pond, at mixing point in the drain and after $700 \mathrm{~m}$ from the mixing point samples of January was observed.

\section{DISCUSSION}

Waste stabilization pond systems are a widely used technique for the treatment of wastewater for rural areas [27]. In this study, settling of solid particulates of wastewater in the septic tanks is one of the main processes to remove organic material from liquid phase 117]. The effluents of the septic tanks are used as influent in WSP. Our results showed that the overall reductions were $44.3 \%$ (TSS), $48.9 \%$ (COD), $50.6 \%$ (BOD), $51.4 \%$ (DP) and $52 \%$ NO3-N. The performance of WSP attained a lower efficiency than expected. The highest efficiency was recorded with anaerobic than facultative and maturation ponds for TSS, COD and BOD reduction.

From the obtained results, it can be observed that the facultative pond was more efficient in the reduction of classical bacterial indicators such as TC, FC and FS. The reduction of classical bacterial indicators was $6 \log$ units for TC, $4 \log _{10}$ units for both FC and E.coli, 5 $\log _{10}$ units for FS and one log unit for coliphage. Additionally, the reduction of salmonellae and Listeria were two log units. The final effluent of maturation was still high in microbial load FC $\left(10^{6}\right)$, E.coli $\left(10^{4}\right)$ $\mathrm{Mpn} / 100 \mathrm{ml}$ and Coliphage (3.1X10) Pfu/100 ml. The final effluent complied with E.coli WHO guidelines for restricted irrigation ${ }^{[34]}$.

Generally Barjenbrach and Erler ${ }^{[5]}$ reported that, there are several causes for deterioration of the purification performance; such as unsuitable design of the pond; incomplete mixing of aerated pond; type of preliminary treatment; insufficient maintenance and increased organic influent loads.

In our study, although, the retention time is sufficient in the ponds, bad removal of BOD, COD and pathogens was observed. The poor removal in maturation pond may be due to some defects in the design of the ponds. The entrance of wastewater to different ponds was from one point. It means bad distribution of the wastewater and bad mixing with the microorganisms in the pond. Also, the increase in the detention time more than recommended may lead to the death of some bacteria and then decrease of the efficiency of the ponds. Modifications of the design of the pond by adding some additional points for entrance of wastewater to the ponds to make complete mix in the different ponds are needed.

The treatment occurring in WSP results from the complex symbiosis of bacteria and algal species which results in an ecological pattern different from that of these organisms grown in pure culture. Changes of $\mathrm{pH}$, temperature and light intensity control the abundance and activity of specific groups of microorganisms in the multi-species microbial communities' characteristic of facultative ponds ${ }^{[24,35]}$.

From the results, $\mathrm{pH}$ values increased from 7.79 to 8.34. It was associated with the increasing algal activity which is expressed as $\mathrm{Chl}$ (a). The increasing in $\mathrm{pH}$ value is due to $\mathrm{CO}_{2}$ consumed during photosynthesis of the algae. The obtained chlorophyll (a) values were as mentioned in literature from $500-2000 \mu \mathrm{g} \mathrm{L} \mathrm{L}^{-1}$ to be occurring in facultative pond ${ }^{[21]}$. Nitrate and phosphate had an inverse relation with $\mathrm{Chl}$ (a), this can be explained by the fact that extensive algal growth exhausts available nutrients. Yan and Jameson, ${ }^{[36]}$ reported that the amount of nitrogen and phosphorus removed from maturation pond depend on algal biomass. Euglena variabilis, Chlamydomonas reinhardii were the most dominant types of green algae that were indication for high organic load ${ }^{[21]}$. 
In this study, the zooplankton community comprises three main classes of phylum Protozoa, phylum Rotatoria, Crustacea (Cladocera, Copepoda and Ostracoda) and larval stages of both Insecta and Nematoda. Protozoa specially Ciliata was the predominant group in all samples taken from the nominated sites and this group was followed by less numbers of rotifers (phylum: Rotatoria). The other two groups of zooplankton, namely, arthropods and larval stages of nematodes were present in few numbers. The dominancy of protozoans indicates the presence of organic pollution. In this respect Ghazy ${ }^{[14]}$ on a study on wastewater of Starch and Glucose Factory showed a positive effect on the majority of protozoan species, more specifically on some ciliates like Paramecium. Also, other species of zooplankton especially amongst cladocerans and rotifers may be used as indicators of organic and chemical pollution ${ }^{[22]}$. These types of pollution were noticed in the studied WSP from the influent and reached maximum in the anaerobic pond as a result of coinciding with protozoans (especially ciliates) peak in this pond and decreased gradually from maturation pond until reached minimum at $700 \mathrm{~m}$ of the effluent mixing point with the agricultural drain water.

On the other hand, it was noticed that zooplankton count and species diversity in influent were less than those in anaerobic pond, facultative pond or maturation pond and this may be due to the presence of other pollutants in this site. These pollutants may be chemicals, pesticides, or toxin-producing strains of fecal bacteria $E$. coli $^{[8]}$.

Chlorophyll "a" measures are included as an approximation of total phytoplankton abundance and also as an indicator of energy inputs into the system through primary productivity. Chlorophyll "a" levels in all sampling sites peak in the facultative pond where the mean zooplankton count /liter $\left(4720 \times 10^{2} \pm 520 \times 10^{2}\right)$ in this site decreased compared with those in the anaerobic pond $\left(28736 \times 10^{2} \pm 40884 \times 10^{2}\right)$ and the maturation pond $\left(8810 \times 10^{2} \pm 11324 \times 10^{2}\right)$. Chlorophyll "a" content in anaerobic and maturation ponds were high (1261.6 and $1333.4 \mu \mathrm{g} \mathrm{L}^{-1}$ ) but decreased than that in facultative pond as a result of grazing of phytoplankton by zooplankton in these two sites. In influent, the chlorophyll "a" content was at its minimum because of high content of suspended solids which obscure light responsible for photosynthesis and the zooplankton count was relatively high in this site in accordance with high counts on ciliates that feed mainly on bacteria and organic matter available in this type of wastewater as indicated from total suspended solids $\left(283 \mathrm{mg} \mathrm{L}^{-1}\right)$.

Thus, zooplankton peak in the anaerobic pond is coincided either with increased green- algal abundance of Clamydomonas, Euglena and Cryptomonas (hence chlorophyll "a" peak), or abundance of bacteria; Muylaert et al. ${ }^{[25]}$ stated that in aquatic ecosystems, bacteria play a key role in the breakdown of organic matter and the remineralization of nutrients. They are grazed upon by protozoa and some metazoans and, as such, from the base of heterotrophic aquatic food chain. Exudates produced by phytoplankton are an important organic substrate for bacteria in many aquatic ecosystems ${ }^{[4]}$. Under oligotrophic conditions, inorganic nutrients may limit bacterial growth ${ }^{[7]}$. Heterotrophic nanoflagellates are often the dominant grazers on bacteria in aquatic ecosystems ${ }^{[29]}$, but specially in eutrophic environments, ciliates can be an important grazers too ${ }^{[19]}$

In this study, it has been noticed that pollution affected species diversity of zooplankton, the number of species in both influent and facultative pond was 10 and 8 species because of high pollution level, whereas in maturation pond increased to 21 species, due to the presence of low pollution levels. Also, pollution affected zooplankton density; pollution in anaerobic pond increased density of ciliates (Protozoa) which are known to be bio-indicators of organic pollution.

Rotaviruses were detected in $62.5 \%$ of influent of WSP. Villena et al. ${ }^{[33]}$ found that rotaviruses were detected in $85.7 \%$ of raw sewage samples in wastewater treatment plants in Cairo. The variation in rotavirus percentage of frequency may be due to increasing of population in Cairo than the population in El-Mofti village. Another reason is that the influent of WSP in this study was the effluent of septic tanks.

The reductions of infectious rotaviruses during WSP processes in this study were $1-2 \log _{10}$ units after anaerobic pond, one $\log _{10}$ after facultative pond and 0-1 $\log _{10}$ after maturation pond. From these results, it can be observed that WSP failed to realize complete removal of infectious rotaviruses. It may be due to the resistancy of rotaviruses to treatment processes. ElSenousy et $a .^{[11]}$ reported that rotaviruses were the most resistant RNA enteric viruses to an activated sludge treatment processes. In this study, the identical sequences of rotaviral VP6 detected in final maturation effluent and in water drain samples after discharge point and after 700 meters of discharge point in January 2006 showed the role of WSP in contaminating drain water with infectious rotaviruses. The absence of infectious rotaviruses before discharge point confirmed this conclusion. Sequencing of amplified products of viral (pathogen) genome may be an evidence to prove the source of contamination of drain water which receives effluents of wastewater treatment plants.

\section{ACKNOWLEDGMENT}

This research was supported by National Research Center in Cairo (project number W.N.0403). We appreciated the technical support of Dr. Badr Hegazy, Civil Division, Faculty of Engineering, Zagazig University, Egypt.

\section{REFERENCES}

1. Abad, F., R. Pintó, C. Villena, R. Gajardo and A. Bosch, 1997. Astrovirus survival in drinking water. Appl. Environ. Microbiol., 63: 3119-3122.

2. APHA, 1998. Standered methodes for the examination of water and wastewater, $20^{\text {th }}$ Edn., American Puplic Healt Association, AWWA, WEF. Washington, D.C.

3. Arar, A., 1988. Background to treatment and use of sewage effluent. In: Treatment and use of Sewage Effluent for Irrigation (eds Pescod M.B. and Arar, A.) Butterworth, Sevenoaks, Kent. 
4. Baines, S. and M. Pace, 1991. The production of dissolved organic matter by phytoplankton and its importance To bacteria-patterns across marine and freshwater system. L. Oceanogr., 36: 1078-1090.

5. Barjenbrach, M. and C. Erler, 2005. A performance review of small German WSPs identifying improvement options. Water Sci. Technol., 51 (12): 43-49.

6. Christian, R., W. Sabine and M. Arnulf, 2003. Acombined system of lagoon and constructed wetland for an effective wastewater treatment. Wat. Res., 37: 2035-2042.

7. Chrzanowski, T., R. Sterner and J. Elser, 1995. Nutrient Enrichment and Nutrient Regeneration Stimulate Bacterioplankton growth. Microb. Ecol., 29: 221-230.

8. Craun, G., F. Berger and R. Calderson, 1997. Coliform Bacteria and Waterborne Disease Outbreaks. J. Am. Water Works Assoc., 89: 96-104.

9. DEV, 1984. Deutsch einheitsverfahren zur wasser, abwasser und schlammuntersuchung, German Standard Methods, Verlag Chemie, Weincheim.

10. Edmondson, W., 1963. Fresh Water Biology. John Wiley And Sons, Inc, New York USA, 2nd Edn.

11. El-Senousy, W., R. Pintó and A. Bosch, 2004. Epidemiology of human enteric viruses in the Cairo water environment. (Paper presented at the 1st International Conference of Environmental Research Division on Sustainable Development Environmental Challenges Facing Egypt. National Research Centre, Cairo, Egypt).

12. EL-Taweel, G., A. Shaban, S. EL-Hawaary and F. ELGohary, 2000. Microbiological Characteristics of Wastewater in Egypt II-Treated Effluent. Egypt J. Microbial., 35: 239-256.

13. Gales, M., E. Julian and R. Kroner, 1966. Method for quantitative determination of total phosphorus in water. Journal of the American Water Works Association, 58: 1361-1368.

14. Ghazy, M., 1990. Impact of Pollution on Zooplankton Communities in certain Segments along the River Nile. M. Sc. Thesis. Faculty of Science. Ain Shams University.

15. Hamzeh, R. and V. Pronce, 2002. Design performance of waste stabilization ponds. mcgrawhill, New York, USA.

16. Kamel, M., 2006. Thermotolerant coliform and Escherichia coli detection and enummeration through multiple tube fermentation. J. Med. Sci., 6 (2): 125-130.

17. Kamel, M. and B. Hgazy, 2006. A Septic Tank System: On Site Disposal J. Applied Sci., 6 (10): 2269-2274.

18. Katzenelson, E., B. Fattal and T. Hostovesky, 1976. Organic flocculation: an efficient second-step concentration method for the detection of viruses in tap water. Appl. Environ. Microbiol., 32: 838-839.

19. Kisand, V. and P. Zingel, 2000. Dominance of ciliate grazing on bacteria during spring in a shallow eutrophic lake. Aquat. Microb. Ecol., 22: 135-142.

20. Lewis, W., 1979. Zooplankton Community analysis. In: Studies on a tropical System. Springer-Verlag,New York Inc, pp: 163.

21. Mara, D. and H. Pearson, 1998. Design manual for waste stabilization ponds in mediterranean countries, Leeds Lagoon Technology International Ltd. Leed, Uk.
22. Mavuti, K. and M. Litterick, 1991. Composition, distribution and ecological role of zooplankton community in lake Victoria, KENYA Warwes. Verh. Int. Verein. Imnol., 24: 1117-1122.

23. McCauley, E., 1984. The estimation of the abundance and biomass of zooplankton in samples. In: A Manual on Methods for the assessment of secondary productivity in Fresh Water. (eds J. A. DowningandF. H. Rigler). Blackwell Scientific, London.

24. Murakani, K., Y. inomari, R. Sudo and Y. Kurihara, 1992. Effect of temperatura on prosperity and decay of genetically engineered micro-organisms in a microcosm system. Water Sci. Technol., 26 (9-11): $2165-2165$

25. Muylaert, K., K. Van Der Gucht, N. Vloemans, L. De Meester, M. Gillis and W. Vyverman, 2002. .Relationship Between Bacterial Community Composition and Bottom-up Versus top-down variables in four Eutrophic Shallow Lakes. Appl. Environ. Microbiol., 68: 4740-4750.

26. Pintó, R., J. Diez and A. Bosch, 1994. Use of the colonic carcinoma cell line CaCo-2 for in vivo amplification and detection of enteric viruses. J. Med. Virol., 44: 310-315.

27. Racault, Y. and C. Boutin, 2005. Waste stabilization ponds in France: state of the art and recent trends. Water Sci. Technol., 51 (12): 1-9.

28. Rose, J., S. Singh, C. Gerba and L. Kelley, 1984. Comparison of microporous filters for concentration of viruses from wastewater. Appl. Environ. Microbiol., 47: 989-992.

29. Sanders, R., K. Porter, S. Bennett and A. Debiase, 1989. Seasonal Patterns of Bacterivory by Flagellates, Ciliates, Rotifers and Cladocerans in a Freshwater Planktonic Community. Limnol. Oceanoger., 34: 673-687.

30. Smith, E. and C. Gerba, 1982. Development of a method for detection of human rotavirus in water and sewage. Appl. Environ. Microbiol., 43: 1440-1450.

31. Starmach, K., 1966. Flora slodkowodna Polska cyanophyta-sinice glaucophyta-glankofity, Tom 2 (Polska Academia NAUK), pp: 85-355.

32. Streble, H. And B. Krauter, 1978. Das leben in wassertropfen. Microflora and microfauna des subasser, Ein Bestimmungsbuch mit 1700 Abbildungen (Stuttgart), pp: 115-197.

33. Villena, C., W. El-Senousy, F. Abad, R. Pintó and A. Bosch, 2003. Group A rotavirus in sewage samples from Barcelona and Cairo: Emergence of unusual genotypes. Appl. Environ. Microbiol., 69: 3883-3891.

34. WHO, 2005. Guidelines for the safe use of wastewater in agriculture 2nd Edn. World Health Organization, Geneva, Switzerland.

35. Wilderer, P., M. Rubio and L. Davids, 1991. Impact of the addition of pure cultures on the performance of mixed culture reactors. Water Res., 25 (11): 1307- 1313.

36. Yan, Y. and G. Jameson, 2004. Application of the Jameson cell technology for algae and phosphorous removal from maturation ponds. Int. J. Miner. Process., 73: $23-28$. 